\title{
GENERAL VERSUS GROUP FACTORS IN MENTAL ACTIVITIES
}

\author{
BY GODFREY H. THOMSON \\ Armstrong College, University of Durham \\ I. Objects of the Paper
}

The objects of this paper are to give a general summary, in non-mathematical language, of various scattered papers which in the writer's opinion prove the invalidity of the reasoning upon which Professor Spearman has based his Theory of General Ability, or Theory of Two Factors: to submit an alternative theory, which may be called the Sampling Theory of Ability, explaining the known facts of mental correlation at least equally as well as does Professor Spearman's theory: and, while admitting that the existence of general ability is still possible, though unproven, to give reasons in support of the greater probability of the Sampling Theory. The two theories are not necessarily exclusive of one another.

\section{Historical}

The controversy as to whether ability in any individual is general, or specific, or in groups or 'faculties' is a very old one, but for the purposes of the present article it is not necessary to go back prior to the above mentioned paper, ${ }^{1}$ the first of a series in which Professor Spearman has developed his Theory of General Ability, or Theory of Two Factors, as it is alternatively named.

Professor Spearman's method in that paper was to measure a number of mental abilities, some of them school subjects, others artificial tests, in a number of persons, and calculate the correlation coefficients of each of these activities with each of the others. These correlation coefficients, he then noticed, had a certain relationship among themselves, a rela-

1 'General Intelligence objectively determined and measured,' C. Spearman, Amer. J. Psychol., 1904, 15, pp. 201-293. 
tionship which may be called hierarchical order, and is explained in detail in the technical papers on the subject. $\mathrm{He}$ saw, quite rightly, that the presence of a general factor would produce this hierarchical order among the coefficients and, reversing this argument, he concluded that the presence of hierarchical order proved the existence of a general factor

A number of experimental researches on these lines, in some of which Professor Spearman himself took part, followed during the next eight years, but with very conflicting results, some experimenters finding the hierarchical order among the coefficients, others finding no such order. Two of the best articles of this period are those of Mr. Cyril Burt, ${ }^{1}$ who found practically perfect hierarchical order, and Dr. William Brown, ${ }^{2}$ who found no trace of such order. The experimental work in each case was psychologically of a high degree of excellence.

Things were in this very unsatisfactory state when an important article by Professor Spearman, in coöperation with Dr. Bernard Hart, appeared in $19120^{3}$ In this article the difficulty of making an unbiased judgment as to the presence or absence of hierarchical order was recognized, and a form of calculation was given for obtaining a numerical criterion of the degree of perfection of hierarchical order, which criterion would be independent of any bias on the part of the calculator. This criterion ranges theoretically from zero, for absence of hierarchical order, to unity, for perfection of hierarchical order. But their formula can, arithmetically, exceed unity.

The authors applied their criterion to all the experimental work available, work dating from various periods, and representing the researches of 14 experimenters on 1,463 men, women, boys and girls. From beginning to end the values of the criterion were positive and very high. The mean was

'Cyril Burt, 'Experimental Tests of General Intelligence,' Brit. J. Psychol., 1909, 3, pp. 94-177.

2 William Brown, 'Some Experimental Results in the Correlation of Mental Abilities,' Brit. J. Psychol., 1910, 3, pp. 296-322.

'General Ability, its Existence and Nature,' by B. Hart and C. Spearman, Brit. J. Psychol., 1912, 5, pp. 51-84. 
almost complete unity. That is to say, Dr. Hart and Professor Spearman claimed that all the data then available showed perfect hierarchical order among the correlation coefficients, even the data of workers like Dr. Brown and Professor Thorndike, who had been unable to detect any such order.

The reasons why the hierarchical order among the correlation coefficients was not obvious at a glance were, according to these authors, two. In the first place, their theory did not entirely deny the presence of group factors of narrow range, and tests which were too similar were, according to them, to be pooled, before the hierarchical order would become apparent. Only in very few cases, however, did they find it necessary to pool tests in the data used. In the second place, the obscuring of the perfect hierarchical order was, according to them, due to the fact that only a small sample of subjects is examined. For this error allowance is made in the formula for calculating their criterion.

Dr. Hart and Professor Spearman therefore considered their 'Theory of Two Factors' proved. This theory considers ability in any activity to be due to two factors. One of these is a general factor, common to all performances. The other is a specific factor, unique to that particular performance, or at any rate extending only over a very narrow range including only other very similar performances. "It is not asserted," they say, "that the general factor prevails exclusively in the case of performances too alike, but only that when this likeness is diminished, or when the resembling performances are pooled together, a point is soon reached where the correlations are still of considerable magnitude, but now indicate no common factor except the general one."

In the same paper Dr. Hart and Professor Spearman consider, and in their opinion confute, two other theories, (a) the older view of Professor Thorndike, viz., a general independence of all correlations, and $(b)$ Professor Thorndike's newer view of 'levels,' or the almost universal belief in 'types.' If the former were true, their criterion would, they consider, show an average value of about zero: if the latter, a low minus value. 
Many experimental researches were inspired by this paper of Dr. Hart and Professor Spearman, of which, as a good example, may be cited one in 1913 by Mr. Stanley Wyatt. ${ }^{1}$ It is I think not too much to say that in practically all of these the application of the Hart and Spearman criterion gave values closely approximating to unity and therefore supporting the Theory of General Ability. But complications began to arise, of which the first of importance will be found in Dr. Edward Webb's monograph on 'Character and Intelligence,' in I9I 5.2 Dr. Webb considered that he had found (in addition to Professor Spearman's General Ability), a second general factor, which he calls 'persistence of motives.' Other writers began to find that their data required for their explanation large group factors, of wider range than those contemplated in the original form of Professor Spearman's theory. ${ }^{3}$ Quite recently Mr. J. C. Maxwell Garnett, discussing the data of a number of workers with the aid of mathematical devices which he has introduced for the purpose, concludes that in addition to the single general factor of Professor Spearman, there are two large group factors which are practically general ${ }^{4}$ (one of them being indeed almost identical with Dr. Webb's second general factor), which he calls respectively 'cleverness' and 'purpose,' both distinct from general ability.

It is clear therefore that in any case the simple original form of Professor Spearman's theory is becoming complicated by additions which tend to modify it very considerably. Meanwhile, however, the present writer had come to the conclusion that the mathematical foundations upon which it was based were in fact incorrect. Before developing the line of argument which led to this, it will be well to re-state Professor Spearman's case in its simplest terms in a few words.

' Stanley Wyatt, 'The Quantitative Investigation of Higher Mental Processes,' Brit. J. Psychol., 1913, 6, pp. 109-133.

'E. Webb, 'Character and Intelligence,'Brit. J. Psychol., Monog. Suppl., 1915, 3, pp. ix and 99 .

${ }^{3}$ See especially E. Carey, 'Factors in the Mental Processes of School Children, Brit. J. Psychol., 1916, 8, pp. 170-182.

'J. C. Marwell Garnett, 'General Ability, Cleverness, and Purpose,' Brit. J. Psychol., 1919, 9, pp. 345-366. 
It is entirely based upon the observation and measurement of hierarchical order among correlation coefficients. It states that after allowance has been made for sampling errors this hierarchical order is found practically in perfection. And it finally states that such a high degree of perfection can only be produced by a general factor, and the absence of group factors, which would mar the perfection.

\section{The Case against the Validity of Professor Spearman's Argument}

It is possible, by means of dice throws or in other ways, to make artificial experiments on correlation, with the immense advantage that the machinery producing the correlation is known, and that therefore conclusions based upon the correlation coefficients can be confronted with the facts. Working on these lines, the present writer made, in 1914, a set of imitation 'mental tests' (really dice throws of a complicated kind) which were known to contain no general factor. The correlations were produced by a number of group factors which were of wide range, and, unlike Professor Spearman's specific or narrow group factors, they were not mutually exclusive.

These imitation mental tests, containing no general factor, gave however a set of correlation coefficients in excellent hierarchical order, and the criterion was when calculated found to be unity, so that had these correlation coefficients been published as the result of experimental work, they would have been claimed by Professor Spearman as proving the presence of a general factor. ${ }^{1}$

In a short reply Professor Spearman laid stress on the fact that this arrangement of group factors which thus produced practically perfect hierarchical order was not a random arrangement, and that it was exceedingly improbable that this one special arrangement should have occurred in each of the psychological researches of many experimenters, so improbable indeed as to be ruled entirely out of court. ${ }^{2}$

1 Godfrey H. Thomson, 'A Hierarchy without a General Factor,' Brit. J. Psychol., I916, 8, pp. 271-281.

2 C. Spearman, 'Some Comments on Mr. Thomson's Paper,' Brit. J. Psychol., 1916,8, p. 282. 
It is clear that Professor Spearman did therefore definitely admit that at any rate one arrangement of group factors existed which would give hierarchical order of sufficient perfection to satisfy completely his criterion. He did more than this, however, for he claimed already to have published, without proof, in an earlier paper, what the effect of a really random overlapping of all the factors in his opinion is, namely that in this case his criterion will be of the same value as the average correlation between the tests. ${ }^{1}$ Now the average correlation between the tests employed in the psychological researches under consideration is not as a rule low. Indeed in those tests which really play an important part in the calculation of the criterion it is usually very high. So that this criterion would, if Professor Spearman's admission be correct, apparently be high on the random overlap theory; that is to say sheer chance would produce considerable though not perfect hierarchical order. This already puts the proof of the Theory of General Ability into a very different position from that which it appeared to occupy immediately after the publication of the paper of Dr. Hart and Professor Spearman in 1912. For in that paper the alternative theories gave values of the criterion which were either zero or negative, and the fact that it actually came out to be almost unity seemed conclusive. But now the comparison is much less definite, for here is a theory which may give high positive values. The criterion must not merely be high and positive to prove the Theory of Two Factors, it must be absolutely unity. True, in Professor Spearman's calculations it does come to unity with most remarkable regularity. But if it can be shown that these calculations are in any way erroneous, then the fact that the comparison is with a theory which can give a high criterion, and not merely with theories which give zero or less, is of great importance.

One reply which Professor Spearman might make to this step of the argument is contained by implication in the footnote on page rog of the already quoted 1914 article in the

${ }^{1}$ C. Spearman, 'The Theory of Two Factors,' Psychol. Rev., I914, 21, p. Iog. See also E. Webb, Character andIntelligence,' Brit. J. Psychol. Monog. Suppl., 1915, No. 3, on page 57 and Appendix, page 82. 
Psychological Review. He appears to think that on the random overlap theory the criterion and the average correlation, though equal, will both be zero or very small on the average. In other words, on this view random overlap will produce hierarchical order if it produces correlation at all, but usually both will be zero. To return to the reply of Professor Spearman to the 'Hierarchy without a General Factor,' the reply namely that this special arrangement would doubtless give such order, but was too improbable to be seriously considered, and that a random arrangement of Group Factors, though it might give some hierarchical order, would not give it in the perfection actually found: the obvious way to find out if this is so or not is to try it, with artificial 'mental tests' formed of dice throws. This the present writer did in November and December of 1918 , after an unavoidable delay of some years. Sets of artificial variables (analogous to the scores in mental tests) were made, in each of which the arrangement of group factors was decided by the chance draws of cards from a pack. ${ }^{1}$ It was found that in every case a very considerable degree of perfection of hierarchical order was produced, quite as high as that found in the correlation data of experimental psychology. A further test was made on that set of data, from among these artificial experiments, which appeared to yield the least perfect hierarchical order. The true values of the correlation coefficients being known, the true degree of perfection of hierarchical order could be correctly calculated, and was 0.59 (perfection being represented by unity). Dice throws were now made to obtain experimental values of the same correlations, and Professor Spearman's criterion applied. As it has done in the case of so many experimental researches in psychology, it gave the value unity. ${ }^{2}$ This set of correlation coefficients, therefore, if it had been published as the result of experiments on mental

${ }^{1}$ Godfrey H. Thomson, 'On the Cause of Hierarchical Order among the Correlation Coefficients of a Number of Varieties taken in Pairs,' Proceedings of the Royal Society of London, 1919, A, 95, pp. 400-408. See also, by the same author, 'The Hierarchy of Abilities,' and 'The Proof or Disproof of the Existence of General Ability,' in Brit. $J$. Psychol., I919, 9, pp. 321-344.

2 Godfrey H. Thomson, 'On the Degree of Perfection of Hierarchical Order among Correlation Coefficients,' Biometrika, 1919, Vol. 12, pp. 355-366. 
tests, would have been claimed by Professor Spearman as additional proof of the existence of a general factor, although in fact there was no such general factor present, and the correlations were due to randomly selected group factors.

The conclusions which appear reasonable from this are (a) that hierarchical order, unless perhaps when it is absolutely perfect, is no proof of the existence of a general factor, and $(b)$ that the Hart and Spearman criterion for hierarchical order is somehow incorrect, and exaggerates the degree of hierarchical order present.

The errors which cause this exaggeration are pointed out in the last cited article in Biometrika, and are mainly two. In the first place, Dr. Hart and Professor Spearman assumed certain quantities to be uncorrelated when they are really strongly correlated, though in a peculiar manner. This error causes the possible values of the criterion to be distributed, not from zero to unity, but from zero to infinity. In the second place, they employ a 'correctional standard' which rejects all the values greater than about $\mathbf{I} \frac{1}{2}$. The possible range for the accepted values is in practice from about $\frac{1}{2}$ to $I \frac{1}{2}$, and their average is naturally about unity. In other words, the remarkable regularity with which this criterion gives the value unity is not a property of the investigated correlation coefficients at all, but is a property possessed by the criterion itself, due to errors and the action of the 'correctional standard.'

In the writer's opinion the work outlined in this section of the present paper finally proves the invalidity of Professor Spearman's mathematical argument in favor of the Theory of Two Factors. If this be so that theory returns to the status of a possible, but unproven, theory.

\section{Hierarchical Order the Natural Order among}

Correlation Coefficients

The fact is that hierarchical order, which Professor Spearman was the first to notice among correlation coefficients, is the natural relationship among these coefficients, on any theory whatever of the cause of the correlations, excepting 
only theories specially designed to prevent its occurrence. It is the absence of hierarchical order which would be a remarkable phenomenon requiring special explanation; its presence requires none beyond what is termed chance.

An analogy from the simple repeated measurements of a linear magnitude may help to illustrate this. Indeed it is rather more than an analogy, being in fact the same phenomenon in its simplest terms and dimensions. It is well known that many measurements of the same quantity, made with all scientific precautions, under apparently the same conditions, and with an avoidance of all known sources of error, nevertheless do not give a number of identical values. The values are all different, but are not without law and order in their arrangement. They are grouped about a center from which the density decreases in both directions, and it is found that this grouping is for most practical purposes closely represented by the Normal or Gaussian Curve of Error. Experimenters are not surprised to find their data obeying the Normal Law, nor do they require a special theory to explain it. On the contrary, it is the departures from the Normal Law which if wide would cause alarm and require special investigation, and if confirmed would require a special theory. In the same way hierarchical order among correlation coefficients should not cause surprise, though any marked variation from this order would demand investigation.

Correlation coefficients are themselves correlated, and $n$ correlation coefficients form an $n$-fold or $n$-dimensional correlation-surface. The particular and convenient form of tabulation of correlation coefficients adopted by Professor Spearman and followed by most other psychological workers brings to light, in the form of "hierarchical order," one of the properties of this correlation-surface of the correlations.

It is true that in the ordinary form of the theory of correlation of correlations, ${ }^{1}$ the variations in the correlation coefficients to which the correlation of these coefficients refers, are variations due to sampling the population; i.e., to taking

1 K. Pearson and L. N. G. Filon, 'On the Probable Errors of Frequency Constants and on the Influence of Random Selection on Variation and Correlation,' Phil. Trans. of the Roy. Soc. London, 1898, 192 A, pp. 299-3II. 
in our case a class of only perhaps 50 English grammar school boys of age 12, instead of all such boys: whereas the hierarchical order we desire to explain is already found in the true 'theoretical' correlation coefficients. This difference is however one of point of view only. It was left partially unexplained in the above cited article ${ }^{1}$ although it was referred to. Further consideration leads to the following resolution of the difficulty.

Suppose that $n$ variates (in our work the scores in mental tests) are so connected by factors that the correlations are all equal and positive. Then let a small sample of the population be taken. The observed correlations will show departures from equality, and will be found to be in hierarchical order. This hierarchical order is due to sampling the population.

Now consider why the correlations do not come out at their true values. They give of course the true values for the sample. The reason of their departing from the true values of the whole population is that (a) some of the factors which really are links between the variates (the mental activities) happen to have remained steadier than usual during the sample. In the limit a factor might happen to retain exactly the same value through the various individuals of the sample. That is, some of the linking factors do not in reality come into action, or not in their full force. (b) On the other hand, some factors which are really different and unconnected may happen by chance to rise and fall together, throughout the sample, and more or less to act as one. That is, fictitious linking factors are created, which would disappear with a larger sample.

Clearly therefore a hierarchy of correlation coefficients, caused by sampling the population, is due to chance having caused a change in the apparent factors acting. It follows that if we make a real change in the factors acting, we shall get a hierarchy, and this is what we do when we choose the mental tests to be employed in any research. Each mental test is a test of a sample of abilities.

The laws governing the correlation of correlation coeffi${ }^{1}$ Godfrey H. Thomson, Proc. Roy. Soc. London, 1919, A, 95, pp. 407 and 408. 
cients which vary because of sampling the population can, in fact, be applied without hesitation to the relationships between 'true' correlations in the whole of any population simply because any such population is itself a sample. English grammar school boys of 12 are themselves a sample of a larger boyhood; the whole human race indeed is a sample of 'what might have been,' selected by the struggle for survival.

The whole question clearly has philosophical bearings on the degree of reality of causal connections; for on this view those chance links in a small sample which were a few paragraphs ago termed 'fictitious links, which would disappear with a larger sample,' do not differ except in degree from the 'real' causal links which we only term real because they persist throughout the largest sample with which we are acquainted.

In another direction there are connections with the difference, which is one of degree only, between what is called 'partial' correlation and 'entire' correlation. ${ }^{\text {' }}$

The conclusion to be drawn from this section of the present paper is that hierarchical order is the natural order to expect among correlation coefficients, on a theory of chance sampling alone, and that therefore, by the principle of Occam's razor, its presence cannot be made the criterion of the existence of any special form of causal connection, such as is assumed in the Theory of Two Factors.

\section{A Sampling Theory of Ability}

In place therefore of the two factors of that theory, one general and the other specific, the present writer prefers to think of a number of factors at play in the carrying out of any activity such as a mental test, these factors being a sample of all those which the individual has at his command.

The first reason for preferring this theory is that of Occam's razor. It makes fewer assumptions than does the

${ }^{2}$ See Karl Pearson, 'On the Infiuence of Natural Selection on the Variability and Correlation of Organs,' Phil. Trans. Roy. Soc. London, 1902, A, 200, pp. I-66.

Godfrey H. Thomson, 'The Proof or Disproof of the Existence of General Ability,' Brit. J. Psychol., 1919, 9, pp. 321-336. 
more special form of theory. It does not deny general ability, for if the samples are large there will of course be factors common to all activities. On the other hand it does not assert general ability, for the samples may not be so large as this, and no single factor may occur in every activity. If, moreover, a number of factors do run through the whole gamut of activities, forming a general factor, this group need not be the same in every individual. In other words general ability, if possessed by any individual, need not be psychologically of the same nature as any general ability possessed by another individual. Everyone has probably known men who were good all round, but Jones may be a good all round man for different reasons from those which make Smith good all round.

The Sampling Theory, then, neither denies nor asserts general ability, though it says it is unproven. Nor does it deny specific factors. On the other hand it does deny the absence of group factors. It is this absence of group factors which is in truth the crux of Professor Spearman's theory, which is not so much a theory of general ability, or a theory of two factors, as a Theory of the Absence of Group Factors. And inasmuch as its own disciples have begun to require group factors to explain their data, its distinguishing mark would appear in any case to be disappearing.

Such group factors as are admitted by Professor Spearman are of very narrow range, and are mutually exclusive, that is they do not overlap. Both these points follow from the sentence used in the 1912 article with Dr. Hart, where it is said that, in the case of performances too alike, "when this likeness is diminished, or when the resembling performances are pooled together, a point is soon reached where the correlations are still of considerable magnitude, but now indicate no common factor except the general one.'

Since this point is soon reached, the group factors must be narrow in range. Since pooling a few performances will obliterate any group factors, they must be exclusive of one another. For if $A, B, C$ and $D$ are four tests, in which $A$ and $B$ have a group factor common to them, and $C$ and $D$ another, 
then of course by pooling $A$ with $B$ and also $C$ with $D$ we can obtain two pools $A B$ and $C D$ which have no link. But if $A, B$ and $C$ have one group factor, and $C$ and $D$ have another then these group factors cannot be separated into specific factors. In fact, a specific factor is a separated group factor, and Professor Spearman's theory asserts that group factors, if any, are separable and mutually exclusive. This is to the present writer the great stumbling block in the way of the acceptance of the Theory of Two Factors, unless 'specific factor' is interpreted in the way suggested later in this article.

It is a fact which will be admitted by most that the same activity is not performed in the same way by different individuals, even though they are equally expert. Not only are specific factors therefore required by this theory for every separate activity, excluding only any which are very closely similar; but also specific factors of different psychological natures are required for each individual. Further, the same individual does not always perform the same activity in the same way. A man using an ergograph will, as he tires, begin to employ muscles other than those naturally used at the outset. When we are returning from a cycle ride muscles are used in a different manner from the style adopted at the start, indeed sometimes deliberate changes are made to give relief. And in the same way a mental task is performed by different methods at different times. Does this then mean a different specific factor for each way of doing a task? All these difficulties appear to argue against the Theory of Two Factors, and seem to the present writer to be considerably cleared up by the Sampling Theory.

Finally, the Sampling Theory appears to be in accordance with a line of thought which has already proved fruitful in other sciences. Any individual is, on the Mendelian theory, a sample of unit qualities derived from his parents, and of these a further sample is apparent and explicit in the individual, the balance being dormant but capable of contributing to the sample which is to form his child. It seems a natural step further to look upon any activity carried out by this 
individual as involving yet a further sample of these qualities.

\section{The Difficulty of 'Transfer of Training'}

Although Professor Spearman's Theory of Two Factors has been chiefly based by him on the line of argument which, it is suggested, has now been proved invalid, viz., the "hierarchy' argument, yet there is another and powerful form of reasoning which can be brought up to its support, based upon the fact that, according to some experimenters, improvement in any activity due to training does not transfer in any appreciable amount to any other activity, except to those very similar indeed to the trained activity. And even those workers who do not agree that this is an experimental fact are usually content to take a defensive attitude and say that transfer is not disproved. Few if any will say that it is proved.

This certainly seems to point to the absence of group factors, and to support Professor Spearman's theory, which only needs to add to itself the assumption that the specific factors are, while the general factor is not, capable of being improved by training, to fit the case admirably. Of course, if transfer really occurs, the argument proves the opposite. And although psychological experiment points on the whole to the absence or the narrowness of transfer, yet popular opinion among business men, schoolmasters, and others is in favor of transfer to a considerable extent. Assuming no transfer, however, how can the Sampling Theory, with its numerous group factors, explain this?

It is necessary to assume that the group factors are all unimprovable or only slightly improvable by training, though they may change with the growth and development of the individual. The improvement which certainly takes place when we practice any activity is due, it may then be assumed, not to improvement in the elemental abilities which form the sample, but to a weeding out, and selection of these. The sample alters, mainly no doubt is diminished, though additions are also conceivable. It becomes a more economical 
sample, and waste of effort in using elements which are unnecessary is avoided. Improvement in any mental activity may on this view be compared with improvement in a manual dexterity, in which it is notorious that the improvement consists largely in the avoidance of unnecessary movements.

When another activity is then attempted, the elemental factors are just the same as they would have been had the practice in the first activity not taken place. The new activity will be performed by a new group of factors, which sample will as in the first case be in the beginning wasteful and will include many unnecessary elements. Transfer of improvement gained in the first activity will therefore not take place except insofar as the second activity is recognized as a mere variant of the original one, in which case the weeding out process which has taken place in the first case may be done at the very first attempt, at any rate to some extent.

To use another analogy, the improvement which takes place when a football team practices playing together for a series of matches is due more to team work than to individual improvement. A new team, even though it contain a large proportion of players from the first team, will not have this unity of action. There will be little transfer of improvement.

According to the view here developed, it is the weeding out of the sample of elemental abilities which is specific. The team work is specific, though the players play for several clubs. This would appear to enable a reconciliation to be affected between the almost universal belief in 'types' of ability (to which Professor Spearman refers) and the experimental facts concerning both correlation and transfer. If there be a general factor at all, it might be the power to shake down rapidly into good team work, in a word, educability. But there seems no objection to assuming that this, instead of being a general factor, is a property of each elemental factor, varying from factor to factor.

To sum up this section: if transfer of training really does not occur to any great extent, then it has to be admitted that 
the Theory of Two Factors readily explains this. But the Sampling Theory can also do so, in a manner which is perhaps not so easy to set forth, but which nevertheless appears to the present writer to be more illuminating and less artificial than the alternative theory.

\section{The 'Faculty Fallacy'}

Since the group factors spoken of in this Sampling Theory are, in the fact that they are supposed to come into play in many different activities, similar to the banished 'faculties' of the mind (though the writer conceives of them as being smaller units than were those faculties) it is probably necessary to defend the theory against the charge of committing what is known as the 'Faculty Fallacy.' This defence is easy. It is only necessary to point out (a) that a person who believes in 'faculties' or 'types' or 'levels' does not necessarily commit the above-mentioned fallacy, and (b) that any charge of being 'faculties' which may be brought against the group factors can of course also be brought against the general factor.

The clearest account of the faculty fallacy known to the writer is given in the older edition of Professor G. F. Stout's 'Manual': "An effect cannot be its own cause, and cannot, therefore, afford its own explanation. But it is a fallacy of not infrequent occurrence to assign as a cause what turns out on examination to be only the effect itself, expressed in different language. ... The classical instance of this confusion is the answer of Molière's physician to the question: 'Why does opium induce sleep?' 'Opium,' he answers, 'produces sleep because it has a soporific tendency.",

Now it is to be clearly noted that there is no logical objection to the physician saying either that opium produces sleep, or that it has a soporific tendency. All that he must avoid doing is to give the one as the cause of the other. And in a similar way there is no logical objection to anyone believing, on the ground of experiment, that if a man has a good memory for historical matters he will, as a fact, have a good memory for all other matters. But if he believes this 
without any other ground than that the name memory is given to these diverse activities, then indeed he is committing the fallacy in question. Even if a man uses the form of words: "Robinson will be a good man for this post, because he has a good memory," he is not necessarily committing any logical fallacy. He may very well mean by this short statement something like the following: "I have noticed that a man who remembers one class of facts well is also frequently good at remembering other classes of facts. I know that Robinson can remember such and such things easily and accurately, therefore $I$ think it very probable that he will be above the average in this job, which requires the memorizing of certain facts." And in this there is no fallacy, whether the conclusion be true or false.

The existence of the group factors spoken of in this paper is deduced with more or less probability from the known experiments. Their existence is an hypothesis which explains these facts, though it is not the only hypothesis to do so. If, as is very probable, the language used in any part of this paper is open to an interpretation which would involve the fallacy, then it can only be said that this is not the interpretation which is intended.

\section{Conclúsions}

Professor Spearman's Theory of Two Factors, which assumes that ability in any performance is due to $(a)$ a general factor and (b) a specific factor (group factors being absent, or at any rate very narrow in range and mutually exclusive) is based chiefly on the observed fact that correlation coefficients in psychological tests tend to fall into 'hierarchical order.' It has been shown, however, that the criterion adopted for evaluating the degree of perfection of hierarchical order present is untrustworthy and has led to overestimation. Such hierarchical order as is actually present is in fact the natural thing to expect, and it is the absence of such which should occasion surprise. The proof of the Theory of Two Factors which is based on the presence of hierarchical order therefore falls to the ground. 'The theory remains a possible 
explanation of the facts but ceases to be the unique explanation. As an alternative theory there is here advanced a Sampling Theory of Ability, in which any performance is considered as being carried out by a sample of group factors. This theory is preferred because it makes fewer and less special assumptions, because it is more elastic and wider, and because it is in closer accord with theories in use in biology and in the study of heredity. 